\title{
SOME FIXED POINT THEOREMS FOR SET VALUED DIRECTIONAL CONTRACTION MAPPINGS
}

\author{
V.M. SEHGAL \\ Department of Mathematics \\ University of Wyoming \\ Laramie, Wyoming 82071 \\ (Received July 5, 1979)
}

ABSTRACT. Let $S$ be a subset of a metric space $X$ and let $B(X)$ be the class of all nonempty bounded subsets of $\mathrm{X}$ with the Hausdorff pseudometric $\mathrm{H}$. A mapping $F: S \rightarrow B(X)$ is a directional contraction iff there exists a real $\alpha \varepsilon[0,1)$ such that for each $x \in S$ and $y \varepsilon F(x), H(F(x), F(z)) \leq \alpha d(x, z)$ for each $z \varepsilon[x, y] \cap S$, where $[x, y]=\{z \varepsilon x: d(x, z)+d(z, y)=d(x, y)\}$. In this paper, sufficient conditions are given under which such mappings have a fixed point. KEY WORDS AND PHRASES: Directional contraction, Hausdorf6 pseudometric. 1980 MATHEMATICS SUBJECT CLASSIFICATION CODES: Primary 47H10, Secondary 54 H25.

1. Introduction.

In this paper, we prove a fixed point theorem for set valued directional contraction mappings (see definition below). The main result extends an earlier result of Assad and Kirk [1] and has some interesting consequences. 
Throughout this paper, $(X, d)$ represents a complete metric space and $B(X)$ is the class of all nonempty bounded subsets of $\mathrm{X}$ with the Hausdorff pseudometric $\mathrm{H}$ induced by $d$ (see [3] p. 33), that is if $A, B \in B(X)$, then

$$
H(A, B)=\underset{a \in A}{\max }\left\{\sup _{a \in A} d(a, B), \sup _{b \in B} d(A, b)\right\} .
$$

It follows immediately from the definition of $H$, that for any $A, B \varepsilon B(X)$,

$$
\begin{gathered}
d(x, B) \leq H(A, B) \text { for any } x \in A, \\
d(x, B) \leq d(x, A)+H(A, B) \text { for any } x \in x,
\end{gathered}
$$

and given $\varepsilon>0$ and $\mathrm{x} \varepsilon \mathrm{A}$, there exists a $\mathrm{y} \varepsilon \mathrm{B}$ such that

$$
d(x, y) \leq H(A, B)+\varepsilon .
$$

For $\mathrm{x}, \mathrm{y} \varepsilon \mathrm{x}$, we will denote

$$
[\mathrm{x}, \mathrm{y}]=\{\mathrm{z} \varepsilon \mathrm{X}: \mathrm{d}(\mathrm{x}, \mathrm{z})+\mathrm{d}(\mathrm{z}, \mathrm{y})=\mathrm{d}(\mathrm{x}, \mathrm{y})\},
$$

and $(x, y]=[x, y] \backslash\{x\},(x, y)=(x, y] \backslash\{y\}$. The following result is due to Caristi [2] and is used in the proof of the main result.

THEORFM (Caristi) Let $\mathrm{f}: \mathrm{X} \rightarrow \mathrm{X}$ be a mapping. If there exists a lower semi-continuous (l.s.c.) mapping $\phi: \mathrm{X} \rightarrow[0, \infty)$ such that for each $\mathrm{x} \varepsilon \mathrm{S}$,

$$
d(x, f(x)) \leq \phi(x)-\phi(f(x)),
$$

then $f$ has a fixed point.

\section{MAIN RESULTS.}

Let $\mathrm{S}$ be a nonempty subset of $\mathrm{X}$.

DEFINITION 1. A mapping $F: S \rightarrow B(X)$ is a directional contraction (d.c)

iff there exists a real $\alpha \varepsilon[0,1)$ such that for each $x \in S$ and $y \varepsilon F(x)$,

$$
H(F(z), F(x)) \leq \alpha d(z, x),
$$

for all $\mathrm{z} \varepsilon[\mathrm{x}, \mathrm{y}] \cap \mathrm{S}$.

The real $\alpha$ in (2.1) will be called a contraction constant of $F$.

THEOREM 1. Let $S$ be a closed subset of $X$ and $F: S \rightarrow B(X)$ be a d.c mapping 
with contraction constant $\alpha$. If $F$ satisfies

a) for each $x \in S, y \varepsilon F(x)>S$, there exists a $z \varepsilon(x, y) \cap S$ with $\mathrm{F}(\mathrm{z}) \subseteq \mathrm{S}$,

b) the mapping $g: S \rightarrow[0, \infty)$ defined by $g(x)=d(x, F(x)$ ) is $\ell . s . c$. ,

then $F$ has a fixed point, that is $x \in F(x)$ for some $x \in S$.

We first prove the following lemma which simplifies the proof of Theorem 1 .

LEMMA. Under the hypothesis of Theorem 1 , for any $\beta, \alpha<\beta<1$, there exists a mapping $A: S \rightarrow B(X)$ with the following properties

i) for each $\mathrm{x} \varepsilon \mathrm{S}, \mathrm{A}(\mathrm{x}) \neq \phi$ and $\mathrm{A}(\mathrm{x}) \subseteq \mathrm{F}(\mathrm{x})$,

ii) if $\mathrm{y} \in \mathrm{A}(\mathrm{x})$, then $\mathrm{d}(\mathrm{x}, \mathrm{y}) \leq(1-\beta+\alpha)^{-1} \mathrm{~d}(\mathrm{x}, \mathrm{F}(\mathrm{x}))$,

iii) if $A(x) \cap S=\phi$ for some $x \in S$, then there exists a $y=y(x) \varepsilon A(x)$ and $a z=z(x, y) \varepsilon(x, y) \cap S$ such that

$$
\mathrm{d}(\mathrm{x}, \mathrm{y}) \leq \mathrm{d}(\mathrm{x}, \mathrm{F}(\mathrm{x}))+(\beta-\alpha) \mathrm{d}(\mathrm{x}, \mathrm{z}) .
$$

PROOF. Define a mapping $A: S \rightarrow B(X)$ by

$$
A(x)=\left\{y \in F(x): d(x, y) \leq(1-\beta+\alpha)^{-1} d(x, F(x))\right\} .
$$

Since $(1-\beta+\alpha)<1, A(x) \neq \phi$ for any $x \varepsilon S$ and satisfies (2.4) and (2.5). Suppose $A(x) \cap S=\phi$ for some $x \in S$. Choose a sequence $\left\{y_{n}\right\} \subseteq F(x)$ such that

$$
\mathrm{d}\left(\mathrm{x}, \mathrm{y}_{\mathrm{n}}\right) \rightarrow \mathrm{d}(\mathrm{x}, \mathrm{F}(\mathrm{x}))
$$

Since the sequence $\left\{y_{n}\right\}$ is eventually in $A(x)$, we may assume that the sequence $\left\{y_{n}\right\} \subseteq A(x)$. It then follows by the supposition that for each $n \varepsilon I$ (positive integers), $y_{n} \varepsilon F(x) \backslash S$ and consequently by (2.2) for each $n \varepsilon I$, there exists a $z_{n}$ satisfying

$$
z_{n} \varepsilon\left(x, y_{n}\right) \cap S \text { and } F\left(z_{n}\right) \subseteq S \text {. }
$$

Now, since $d\left(x, z_{n}\right) \leq d\left(x, y_{n}\right)$, it follows by $(2.7)$ that there is a subsequence $\left\{z_{n_{k}}\right\}$ of the sequence $\left\{z_{n}\right\}$ and a real $\lambda \geq 0$ such that

$$
\mathrm{d}\left(\mathrm{x}, \mathrm{z}_{\mathrm{n}_{\mathrm{k}}}\right) \rightarrow \lambda
$$


We claim that $\lambda>0$. Suppose $\lambda=0$. Then the sequence $\left\{z_{n_{k}}\right\} \rightarrow x$. Moreover, since $y_{n} \varepsilon F(x)$, it follows by the definition of $F$ and (2.8) that

$$
H\left(F(x), F\left(z_{n_{k}}\right)\right) \leq \alpha d\left(x, z_{n_{k}}\right) \rightarrow 0 \text { as } k \rightarrow \infty \text {. }
$$

Now, (2.10) implies that $F(x) \subseteq S$, for if $y$ is an arbitrary element of $F(x)$, then by (1.3) for each $k \in I$, there is a $w_{k} \in F\left(z_{n_{k}}\right)$ such that $d\left(y, w_{k}\right) \leq H\left(F(x), F\left(z_{n_{k}}\right)\right)+\frac{1}{k} \rightarrow 0$ as $k \rightarrow \infty$. Since $\left\{w_{k}\right\} \subseteq S$ and $s$ is closed, it follows that $\mathrm{y}$ and hence $\mathrm{F}(\mathrm{x}) \subseteq \mathrm{S}$. However, this contradicts the supposition that $\mathrm{A}(\mathrm{x}) \cap \mathrm{S}=\phi$. Thus $\lambda>0$. Now choose an $\varepsilon>0$ such that $\delta=(\beta-\alpha) \lambda-\varepsilon>0$. Then by (2.9), $(\beta-\alpha) \mathrm{d}\left(\mathrm{x}, \mathrm{z}_{\mathrm{n}_{\mathrm{k}}}\right) \geq \delta$ eventually and hence by (2.7) and the last inequality,

$$
\mathrm{d}\left(\mathrm{x}, \mathrm{y}_{\mathrm{n}_{\mathrm{k}}}\right) \leq \mathrm{d}(\mathrm{x}, \mathrm{F}(\mathrm{x}))+\delta \leq \mathrm{d}(\mathrm{x}, \mathrm{F}(\mathrm{x}))+(\beta-\alpha) \mathrm{d}\left(\mathrm{x}, \mathrm{z}_{\mathrm{n}_{\mathrm{k}}}\right)
$$

eventually. Thus there exists a $y=y_{n_{k}}$ and the corresponding $z=z_{n_{k}}$ satisfying (2.8) such that (2.6) holds.

PROOF OF THEOREM 1 . Define a mapping $\mathrm{f}: \mathrm{S} \rightarrow \mathrm{S}$ as follows: for $\mathrm{x} \varepsilon \mathrm{S}$, let $f(x)$ be any element of $A(x) \cap S$ if $A(x) \cap S \neq \phi$; and if $A(x) \cap S=\phi$, then by the lemma, there exist elements $y=y(x) \varepsilon A(x)$ and $z=z(x, y) \varepsilon(x, y) \cap S$ satisfying (2.6), let $f(x)=z$ in this case. Note that for any $x \in S$,

$$
H(F(x), F(f(x)) \leq \alpha d(x, f(x)) .
$$

This is obvious if $A(x) \cap S=\phi$ and if $A(x) \cap S \neq \phi$, then since $f(x) \in F(x)$ and $f(x) \varepsilon[x, f(x)] \cap S$, therefore the definition of $F$ implies (2.11). Set $\phi(x)=(1-\beta)^{-1} g(x)$. Then $\phi$ is l.s.c. on $s$. We show that $f$ satisfies $(1.4)$. Let $x \in S$. We consider cases (i) when $A(x) \cap S \neq \phi$ and case (ii) when $A(x) \cap S=\phi$. In case (i), $f(x) \varepsilon A(x)$ and hence by (2.5), $d(x, f(x)) \leq(1-\beta+\alpha)^{-1} d(x, F(x))$. This implies that $\alpha(1-\beta)^{-1} d(x, f(x)) \leq \phi(x)-d(x, f(x))$. Therefore, by (1.1), (2.11) and the last inequality

$$
\phi(f(x))=(1-\beta)^{-1} g(f(x)) \leq(1-\beta)^{-1} H(F(x), F(f(x))) \leq \phi(x)-d(x, f(x)) .
$$


Thus (1.4) holds in this case. In case (ii), there is a $y=y(x) \in F(x)$ such that $f(x) \varepsilon(x, y)$ and satisfies (2.6). Thus by (2.6), $d(f(x), F(x)) \leq d(f(x), y)=d(x, y)-d(x, f(x)) \leq d(x, F(x))-(1-\beta+\alpha) d(x, f(x))$. It now follows by (1.2) and (2.11) and the above inequality that $(1-\beta) \phi(f(x))=g(f(x)) \leq d(f(x), F(x))+H(F(x), F(f(x))) \leq d(x, F(x))-(1-\beta) d(x, f(x))$, that is

$$
d(x, f(x)) \leq \phi(x)-\phi(f(x)) .
$$

Thus $f$ satisfies (1.4) and consequently by Caristi's theorem $f(x)=x$ for some $x \in S$. This implies that $x \in F(x)$ for otherwise $f(x) \notin A(x) \cap S$ and hence by the definition of $f, A(x) \cap S=\phi$. Thus $f(x) \varepsilon(x, y(x))$ for some $y(x) \varepsilon A(x)$. This contradicts $x \neq f(x)$. Consequently, $x \in F(x)$.

Recall, that a metric space is called convex iff for each $x, y \varepsilon x, x \neq y$ there exists a $z \in(x, y)$. It is easy to show (see [4]) that if $S$ is a iclosed subset of a complete, convex metric space and $\mathrm{x} \varepsilon \mathrm{S}$ and $\mathrm{y} \notin \mathrm{S}$, then there exists a $z \varepsilon[x, y) \cap \partial S$ where $\partial S$ denotes the boundary of $S$. As a result of this, the following is an immediate consequence of Theorem 1.

COROLLARY 1. Let $X$ be convex and $S$ a closed subset of $X$. Let $F: S \rightarrow B(X)$ be a d.c mapping such that $f(\partial S) \subseteq S$. If $g(x)=d(x, F(x))$ is l.s.c. on $s$, then $F$ has a fixed point.

The following special case of Corollary 1 extends to $B(X)$ an iearlier result of Assad and Kirk [1].

COROLLARY 2. Let $X$ be convex and $S$ a closed subset of $X$. Suppose $F: X \rightarrow B(X)$ satisfies the condition: there exists an $\alpha \varepsilon[0,1)$ such that for all $\mathrm{x}, \mathrm{y} \in \mathrm{S}$,

$$
H(F(x), F(y)) \leq \alpha d(x, y) .
$$

If $F(\partial S) \subseteq S$, then $F$ has a fixed point. 
PROOF. Since a mapping $F$ satisfying (2.12) is a d.c mapping, it suffices to show that the mapping $g$ on $S$ defined by $g(x)=d(x, F(x))$ is continuous. To prove this, let $\left\{x_{n}\right\}$ be a sequence in $S$ such that $\left\{x_{n}\right\} \rightarrow x \varepsilon S$. It follows that for each $n \varepsilon I$,

$$
g(x)=d(x, F(x)) \leq d\left(x, x_{n}\right)+d\left(x_{n}, F(x)\right) \leq d\left(x, x_{n}\right)+g\left(x_{n}\right)+H\left(F\left(x_{n}\right), F(x)\right) .
$$

That is, $g(x) \leq g\left(x_{n}\right)+(1+\alpha) d\left(x_{n}, x\right)$. Similarly, it follows that for each $\mathrm{n} \varepsilon \mathrm{I}, \mathrm{g}\left(\mathrm{x}_{\mathrm{n}}\right) \leq \mathrm{g}(\mathrm{x})+(1+\alpha) \mathrm{d}\left(\mathrm{x}_{\mathrm{n}}, \mathrm{x}\right)$. Thus $\left|\mathrm{g}\left(\mathrm{x}_{\mathrm{n}}\right)-\mathrm{g}(\mathrm{x})\right| \rightarrow 0$ as $\mathrm{n} \rightarrow \infty$.

\section{REFERENCES}

1. Assad, N. A. and W. A. Kirk. Fixed point theorems for set valued mappings, Pacific J. of Mathematics 43 3(1972) 553-561.

2. Carist1, J. Fixed point theorems for mappings satisfying inwardness conditions, Trans. Amer. Math. Soc. 215(1976) 241-251.

3. Kelly, J. L. and I. Namioka. Linear Topological Spaces, D. Van Nostrand, Princeton, N.J., 1963.

4. Sehgal, V. M. and C. H. Su. Some fixed point theorems for nonexpansive mappings in locally convex spaces, Bull. U.M.I. (4) $10(1974)$ 598-601. 


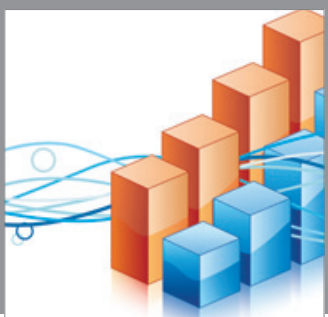

Advances in

Operations Research

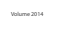

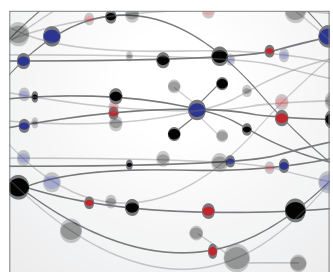

\section{The Scientific} World Journal
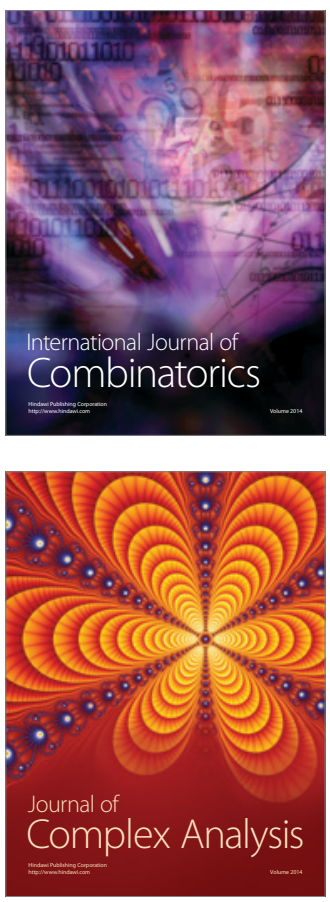

International Journal of

Mathematics and

Mathematical

Sciences
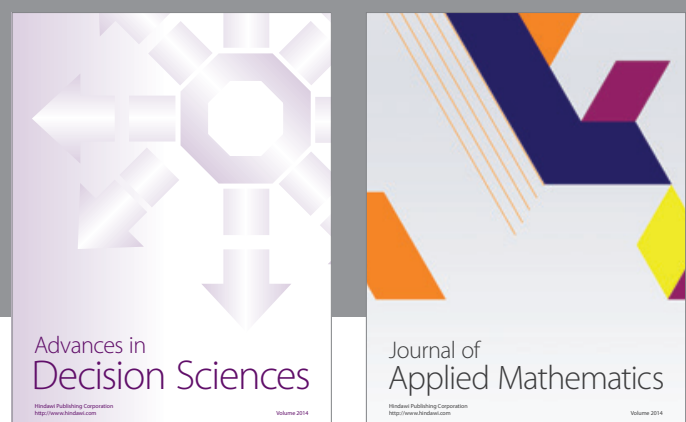

Journal of

Applied Mathematics
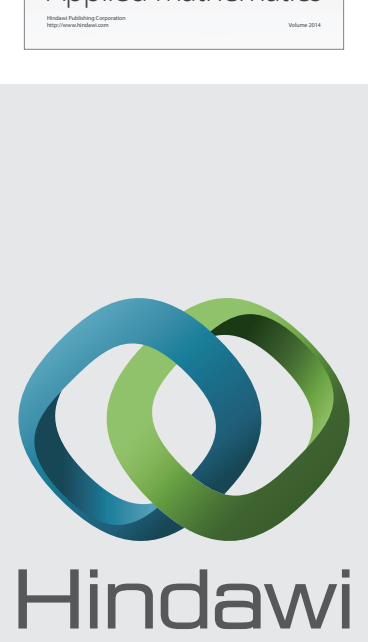

Submit your manuscripts at http://www.hindawi.com
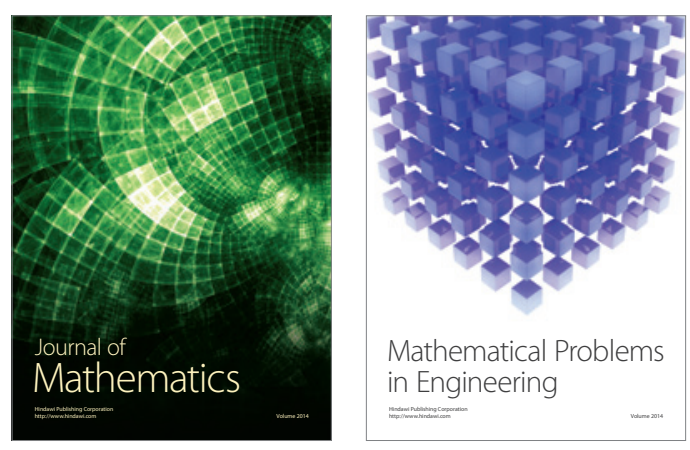

Mathematical Problems in Engineering
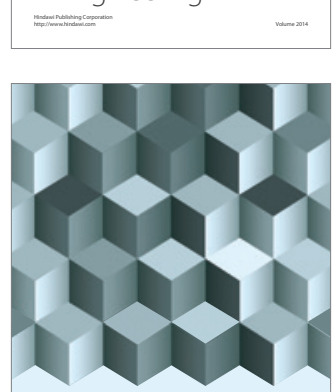

Journal of

Function Spaces
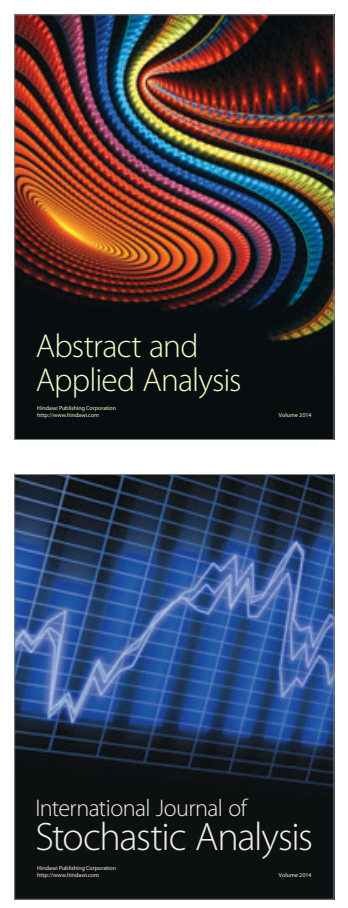

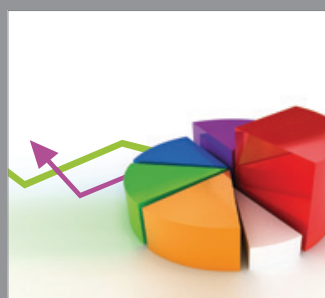

ournal of

Probability and Statistics

Promensencen
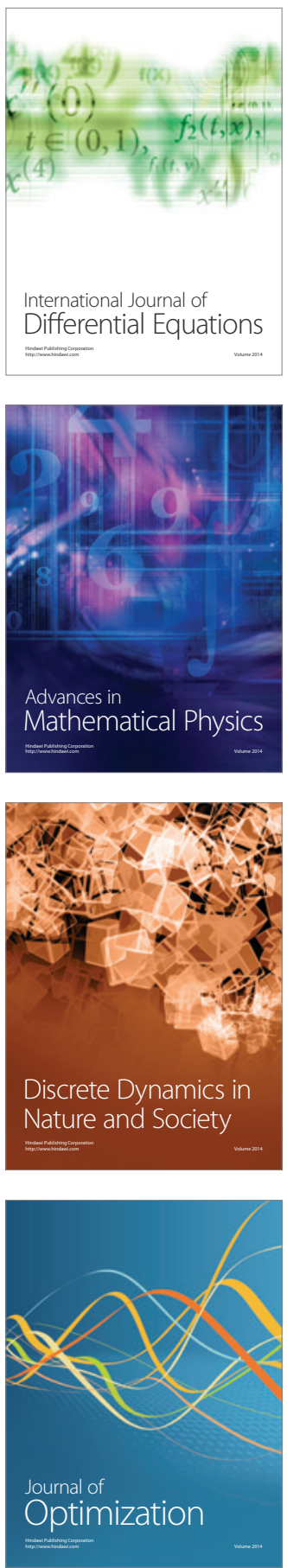